\title{
Depressão, ansiedade e psicopatia: um estudo correlacional com indivíduos privados de liberdade
}

Depression, anxiety and psychopathy: a correlational

study with persons deprived of liberty

Fernanda de Vargas', Fernanda Xavier Hoffmeister', Priscila Flores Prates', Silvio José Lemos Vasconcellos

\section{RESUMO}

Objetivo: Investigar se existe correlação entre sintomas depressivos, sintomas ansiosos e psicopatia em 25 prisioneiros de um município do Rio Grande do Sul. Métodos: Para a coleta de dados, foram utilizados os Inventários de Depressão e Ansiedade Beck e a Escala Hare para psicopatia. As entrevistas foram realizadas nas dependências de uma instituição prisional de forma individual. Resultados: Foi encontrada correlação estatisticamente significativa entre depressão e ansiedade, e o escore total de psicopatia não se correlacionou com ansiedade, somente com depressão. Por outro lado, o fator 2 da escala, referente ao aspecto comportamental do transtorno, apresentou correlação com ansiedade e depres-

\section{Palavras-chave}

Depressão, ansiedade, psicopatia, prisioneiros.

\section{Keywords}

Depression, anxiety, psychopathy, prisoners. são. Conclusão: Embora alguns dados tenham sido concordantes com os da literatura, a pesquisa apresentou resultados não encontrados em estudos anteriores. Dessa forma, evidencia-se a necessidade de realizar novos estudos na área.

\section{ABSTRACT}

Objective: Evaluate the levels of correlation between depression, anxiety and psychopathy in 25 prisoners in a city of Rio Grande do Sul. Methods: To collect the data, we used Beck Depression Inventory and Beck Anxiety Inventory as well as the Hare Psychopathy Scale. The interviews were conducted individually in a prison house. Results: We found statistically significant correlation between depression and anxiety, and the total score of psychopathy was not correlated with anxiety, only with depression. On the other hand, the factor 2 of the scale, related to the behavioral aspect of the disorder, correlated with anxiety and depression. Conclusion: Although some data were consistent with the literature, this research has shown results which were not obtained in previous studies. Thus, it highlights the need for further studies in the area.

1 Universidade Federal de Santa Maria (UFSM).

Endereço para correspondência: Fernanda de Vargas Universidade Federal de Santa Maria (UFSM), Santa Maria, RS, Brasil E-mail:vargasfezinha@gmail.com 


\section{INTRODUÇÃO}

As alterações no humor são comuns na maioria dos sujeitos. Contudo, quando existem frequência e intensidade que possam resultar em sofrimento e prejuízos pessoais e sociais, essas alterações podem ser consideradas patológicas'. Dessa forma, os transtornos depressivos podem implicar significativos prejuízos no funcionamento do sujeito, entre eles diminuição da autoestima e desesperançąa, Esses transtornos são considerados um problema de saúde pública de acordo com variáveis como diminuição de tempo de trabalho, diminuição da qualidade de vida, custos referentes à assistência médica, implicações na família, entre outras ${ }^{46}$.

Já os transtornos de ansiedade caracterizam-se pelas sensações de medo e ansiedade excessiva e perturbação comportamental. O que diferencia os transtornos de ansiedade do medo e da ansiedade adaptativos e necessários à sobrevivência é a intensidade, pois são sentimentos excessivos e persistem além do período apropriado ao nível de desenvolvimento humano. Sendo assim, sinalizam, da mesma forma que os transtornos depressivos, prejuízos pessoais e sociais para os indivíduos?

Embora poucos estudos investiguem os transtornos depressivos e os transtornos de ansiedade em homens e em populações minoritárias como a de indivíduos em situação de aprisionamento ${ }^{8}$, diversos fatores presentes no contexto carcerário reforçam a hipótese de que essa população apresenta índice elevado de sintomas depressivos. Fatores como privação do convívio social, privação de liberdade, estrutura rígida e de controle sobre os sujeitos, mudança de ambiente e situações de tensão poderiam contribuir para a incidência de transtornos de ansiedade e transtornos depressivos 5 .

Por conseguinte, torna-se relevante realizar um estudo que consiga avaliar esses transtornos e suas implicações. Nesse sentido, justifica-se a realização de um estudo que busque investigar também a associação entre os transtornos depressivos e de ansiedade com a psicopatia, já que estudos atuais evidenciam uma associação entre algumas características da psicopatia e o suicídio9,10

A literatura da área destaca, ainda, a associação entre psicopatia e baixa ansiedade, ou seja, psicopatas possuem déficits em respostas emocionais relacionadas à ansiedade, diferentemente de indivíduos sem o transtorno ${ }^{11-13}$. Nesse sentido, um dos modelos que busca explicar essa fraca associação é o modelo dual-déficit, que sugere que indivíduos com o transtorno psicopático, quando colocados em situações de risco, apresentam baixa ansiedade social devido a um funcionamento deficitário no behavioral inhibition system (BIS). Essa compreensão coaduna-se com pressupostos da Psicologia Evolucionista, uma vez que níveis mais baixos de ansiedade poderiam contribuir para uma maior manifestação de dominância nos nichos sociais nos quais a espécie se desenvolveu ${ }^{14}$.
O presente estudo objetivou investigar se existe correlação entre sintomas depressivos, sintomas ansiosos e psicopatia entre apenados que cumprem pena em regime fechado, em uma instituição prisional de um município do Rio Grande do Sul. Acredita-se que os resultados podem auxiliar no que se refere à proposta de colaborar com o trabalho dos profissionais que atuam nesses locais, possibilitando, assim, intervenções precoces e tratamentos adequados a essa população.

\section{MÉTODOS}

A presente pesquisa caracteriza-se como transversal e quantitativa; a pesquisa quantitativa permite testar teorias objetivas, examinando a relação de variáveis. Essas variáveis podem ser medidas por meio de instrumentos, possibilitando a análise dos dados numéricos mediante procedimentos estatísticos ${ }^{15}$. O estudo transversal é caracterizado pela limitação do tempo, ou seja, a coleta dos dados é realizada em determinado momento, sem se prolongar por longo período ${ }^{16}$.

Participaram da pesquisa 25 apenados, maiores de 18 anos, do sexo masculino, que cumprem pena em regime fechado em uma penitenciária de um município do estado do Rio Grande do Sul. A média de idade foi de 33,4 (DP = 8,9). Identificou-se que a maioria dos apenados, 18 deles, apresentava baixa escolaridade (ensino fundamental incompleto), 15 eram solteiros e 10 já haviam se envolvido em mais de três crimes. Ainda sobre a questão jurídica dos apenados, identificou-se que 18 deles eram reincidentes, e somente sete eram réus primários.

Nenhum dos participantes estava em processo de avaliação para progressão de pena durante a realização da coleta de dados, nem apresentou sintomas psicóticos, de acordo com testagem específica. Eram sujeitos alfabetizados, de forma que conseguiram ler e assinar o Termo de Consentimento Livre e Esclarecido (TCLE).

De acordo com os objetivos do estudo, foram aplicados os instrumentos:

- Mini-International Neuropsychiatric Interview (MINIPlus): questionário breve, compatível com os critérios do DSM-III/IV e da CID-10, que se configura como uma entrevista diagnóstica para transtornos mentais, sendo organizada por módulos independentes. Nesse estudo, foi aplicado o Módulo Transtornos Psicóticos para a verificação de presença dos sintomas ou não ${ }^{17}$.

- Escala Hare (Hare Psychopathy Checklist-Revised P(L-R): desenvolvida para avaliação da psicopatia em contexto carcerário. O instrumento consiste em uma entrevista semiestruturada com o participante, mensurando características afetivas, interpessoais e comportamentais. Configura-se como uma escala psicométrica composta por 20 itens pontuados por 
meio de uma escala ordinal de três pontos ( 0 - ausente; 1 - parcialmente presente; 2 - presente), considerando, para tanto, o grau com que o comportamento e a personalidade do avaliando se equiparam à descrição apresentada no manual18,19.

- Escala Beck de Depressão (BDI): escala de autorrelato, a qual inclui alternativas que descrevem como o indivíduo está se sentindo no dia da administração do instrumento e, também, na última semana, para que possam ser percebidos traços mais persistentes. Contém 21 itens, cada um com quatro alternativas, de acordo com graus crescentes dos sintomas de depressão ${ }^{20}$.

- Escala Beck de Ansiedade (BAI): escala de autorrelato, constituída por 21 itens que apresentam afirmações que descrevem sintomas de ansiedade. O sujeito responde em relação à presença de cada sintoma: absolutamente não, levemente, moderadamente, gravemente, constituindo, assim, uma série escalar de 0 a 3 pontos ${ }^{20}$.

Salienta-se que foi realizada consulta à equipe técnica da instituição citada (assistentes sociais, psicólogas e advogado), bem como as triagens/prontuários dos apenados, com intuito de verificar algumas informações. A consulta aos prontuários foi realizada com o objetivo de verificar a situação jurídica dos participantes, ou seja, foram coletadas informações como delito, presença ou não de regressão de pena, reincidência criminal e data prevista para a progressão de pena.

A consulta às psicólogas deu-se com intuito de verificar se os níveis de depressão e ansiedade encontrados nos instrumentos também eram observados nas interações clínicas dos apenados que recebiam atendimento psicológico na penitenciária. No que se refere à Escala Hare, a consulta foi realizada tanto com as psicólogas quanto com as assistentes sociais e o advogado, pois o próprio instrumento prevê a coleta de informações colaterais de diferentes fontes, como familiares, equipe institucional e prontuários ${ }^{18,19}$. Para a realização da referida pesquisa, o projeto foi submetido à entidade responsável pelas casas prisionais do estado do Rio Grande do Sul, que autorizou a realização da coleta de dados nas dependências da penitenciária. Posteriormente, o projeto foi enviado e aprovado pelo Comitê de Ética da Universidade à qual os pesquisadores estão vinculados. Como a pesquisa envolveu seres humanos, ela respeitou os preceitos que norteiam tais estudos, conforme descrito nas Diretrizes e Normas Regulamentadoras de Pesquisa envolvendo Seres Humanos (Resolução no 466/12 do Conselho Nacional de Saúde) $)^{21}$.

Somente após a aprovação do Comitê de Ética (Parecer no 718.898), foi realizado contato com a direção da referida casa prisional para combinar horários e procedimentos a serem adotados durante a pesquisa. Dessa forma, com auxílio do advogado da instituição, foi realizada uma primeira triagem, de acordo com os critérios de inclusão/exclusão, para a seleção dos participantes. Em um segundo momento, os apenados selecionados foram chamados individualmente e, a eles, foram explicados todos os preceitos éticos que envolviam a pesquisa, bem como seus objetivos, além da leitura do TCLE.

Aqueles que aceitaram participar assinaram o termo e, então, a coleta iniciou-se.

A aplicação dos instrumentos durou em média 90 minutos e ocorreu nas dependências da penitenciária. Esse procedimento foi realizado até ser alcançado o número de 25 participantes. Para a realização da análise dos dados, foi utilizada estatística descritiva. Após a realização do Teste de Normalidade (Kolmogorov-Smirnov Test), optou-se por utilizar a correlação de Pearson.

\section{RESULTADOS}

No que concerne à pontuação nas Escalas Beck e na Escala Hare, e a respeito da pontuação nos fatores 1 e 2 do PCL-R, a tabela 1 apresenta os principais resultados. Salienta-se que, em relação especificamente aos fatores do PCL-R, foi utilizada a estrutura de dois fatores. Nesse modelo, o fator 1 representa os sintomas emocionais e interpessoais do transtorno, e o fator 2 está relacionado aos sintomas do desvio social desses indivíduos, seu estilo de vida²2.

Tabela 1. Médias das pontuações nos instrumentos e consistência interna

\begin{tabular}{lccc}
\hline Escalas & $M(n=25)$ & DP & Alpha de Cronbach \\
\hline BDI & 12,7 & 8,8 & 0,82 \\
BAI & 7,7 & 7,4 & 0,84 \\
PCL-R & 18,6 & 8,1 & 0,89 \\
\hline
\end{tabular}

M: média; DP: desvio-padrão.

Referente à presença de sintomas de psicopatia, a pesquisa possibilitou identificar quatro apenados com sintomas do transtorno, considerando pontuação 30 para o ponto de corte do $\mathrm{PCL}-\mathrm{R}^{18}$. Ao analisar a tabela 2, observa-se que o nível mínimo para sintomas depressivos é mais frequente entre os participantes, seguido do nível leve, moderado; somente um participante apresentou o maior nível (grave). No que se refere às frequências na escala de transtornos de ansiedade, observa-se, da mesma forma, maior número de sujeitos com nível mínimo, entretanto nenhum apresentou nível grave.

A respeito das manifestações relacionadas aos quadros e seu tratamento, dentro da instituição onde a pesquisa foi realizada, destaca-se que, após consulta à equipe técnica, se verificou que, dos 25 participantes, 17 não estavam tendo acompanhamento psicológico no período em que a pesqui- 
sa foi realizada. Dos oito sujeitos que eram acompanhados por psicólogas, quatro apresentavam sintomas depressivos ou ansiosos, de acordo com as psicólogas responsáveis pelos atendimentos. Os demais recebiam atendimentos por outras demandas. Desses pacientes identificados com sintomas, dois apresentaram sintomas depressivos durante a entrevista, e um deles apresentou sintomas ansiosos em nível moderado. A partir das correlações realizadas, observou-se uma correlação positiva, estatisticamente significativa, entre sintomas depressivos e ansiosos. O presente estudo também encontrou correlação positiva, estatisticamente significativa, entre os escores totais de psicopatia e de transtorno depressivo. A correlação entre transtornos de ansiedade e psicopatia foi fraca e sem significância.

É possível observar na tabela 3 que os escores totais do $\mathrm{BDI}$ e BAI não se correlacionaram de forma significativa com os escores referentes ao fator 1 da Escala Hare. No entanto, os escores do fator 2 da Escala Hare apresentaram correlação estatisticamente significativa com os escores das escalas que avaliam sintomas de transtornos depressivos e sintomas de transtornos ansiosos. Não houve correlação significativa entre o fator e os sintomas de depressão quando controlada a variável ansiedade ( $r=0,32 ; p>0,05)$, não havendo também correlação significativa entre o citado fator e os sintomas de ansiedade quando controlada a variável depressão $(r=-0,13$; $P>0,05)$.

Tabela 2. Número de participantes em cada nível de sintomas nas Escalas Beck

\begin{tabular}{lll}
\hline Nível & BDI & BAl \\
\hline Mínimo & 13 & 18 \\
Leve & 09 & 04 \\
Moderado & 02 & 03 \\
Grave & 01 & 00 \\
Total & 25 & 25 \\
\hline
\end{tabular}

Fonte: Dados coletados pelos autores.

Tabela 3. Correlações dos escores nos instrumentos

\begin{tabular}{lcc}
\hline & BDI & BAl \\
\hline BDI & - & $0,689^{* *}$ \\
PCL-R & $0,447^{*}$ & 0,256 \\
Fator 1 & 0,239 & 0,100 \\
Fator 2 & $0,594^{* *}$ & $0,428^{*}$ \\
\hline${ }^{*} p<0,05 ;{ }^{* *} p<0,01$. & &
\end{tabular}

\section{DISCUSSÃO}

No que se refere ao diagnóstico de psicopatia, os resultados citados no presente estudo concordam com a literatura científica relacionada à temática. De acordo com Patrick²3, no contexto carcerário, estima-se um percentual de 15\% a 25\% de diagnósticos do transtorno. Uma pesquisa internacional que ratifica esses achados encontrou um percentual de $20,5 \%$ de psicopatia entre os participantes ${ }^{31}$.

Os resultados do presente estudo não demonstraram um número significativo de participantes com sintomas depressivos, nem com sintomas de ansiedade nesse mesmo contexto. Esses resultados se assemelham muito aos encontrados na pesquisa de Araújo et al. ${ }^{8}$, a qual investigou a ocorrência de transtornos depressivos e transtornos de ansiedade em 60 presidiários. Os autores encontraram baixos níveis dos transtornos entre os participantes.

Por outro lado, a pesquisa de Tavares et al..$^{24}$ encontrou um índice significativo de presidiários com transtorno depressivo maior (63\%). Outra pesquisa que vai ao encontro desse resultado é a de Perez et al..$^{25}$, realizada com 34 indivíduos encarcerados na Colômbia, que encontrou dados indicativos de alta prevalência de sintomas depressivos em população carcerária (56\% de indivíduos evidenciando a presença de sintomas moderados a severos). Outro estudo internacional que corrobora esses achados é o de Stinson et al. ${ }^{26}$. As autoras aplicaram instrumentos para verificação dos sintomas depressivos e ansiosos em 68 criminosos sexuais e encontraram um percentual de 35\% de participantes com sintomas depressivos clinicamente significativos e $22 \%$ de criminosos com sintomas de ansiedade.

Gardner e Bell127 assinalam que os sintomas dos transtornos de ansiedade e dos transtornos depressivos podem aparecer em circunstâncias reais de ameaça, como também em outros momentos, estando relacionados a alterações ambientais, como mudanças econômicas e sociais. No entanto, a partir dos resultados do presente estudo, é possível inferir que os fatores de risco apresentados na literatura parecem não ser determinantes para o desenvolvimento de transtornos depressivos e ansiosos nessa população, e sim a forma como esses indivíduos interpretam os acontecimentos/eventos de sua vida.

Essa afirmação equivale a dizer que, embora exista influência do meio, o fator que mantém o transtorno começa no indivíduo, ou seja, na sua forma distorcida de perceber a si próprio, o ambiente e o futuro. Essa postulação vai ao encontro da teoria de Aaron Beck, que afirmou que, na infância, são desenvolvidos esquemas cognitivos (distorções cognitivas e pensamentos automáticos), que poderiam predispor o indivíduo a interpretar de forma negativa os eventos vivenciados em seu cotidiano ${ }^{28}$.

Conforme Fonseca ${ }^{29}$, é alta a probabilidade de identificar, em penitenciárias, sujeitos com transtornos mentais que não recebem acompanhamento adequado, mesmo que antes da situação de privação e liberdade esses sujeitos tenham realizado algum tratamento para transtornos depressivos, ansiosos, entre outros. Assim, ressalta-se a importância do atendimento psicológico dentro de contextos prisionais. 
Acerca da associação encontrada entre sintomas depressivos e ansiosos, uma pesquisa realizada pela Associação de Transtornos de Ansiedade da América ${ }^{30}$ mostrou que 50\% dos pacientes que foram diagnosticados com transtornos de ansiedade também foram diagnosticados com transtornos depressivos. Embora sejam distúrbios diferentes, os sintomas podem aparecer de forma conjunta. Nesse sentido, outros estudos confirmam esses achados, encontrando forte relação entre os sintomas dos transtornos ${ }^{31,32}$.

O trabalho de Torkelsen e Myklebust ${ }^{33}$ não encontrou associação entre psicopatia e transtornos depressivos nem entre psicopatia e transtornos de ansiedade em uma população de detentos, embora os autores tenham utilizado outras escalas para investigar a associação entre o transtorno psicopático e afetividade negativa. Por outro lado, a pesquisa de Stinson et al. ${ }^{26}$ evidenciou que $26,5 \%$ dos participantes apresentavam psicopatia e também características depressivas, assim como 15\% dessa mesma população investigada apresentaram psicopatia e sintomas consistentes com um diagnóstico de transtorno de ansiedade.

O presente trabalho, considerando o tamanho da amostra investigada, a sua heterogeneidade e os instrumentos utilizados, não objetivou testar o assim chamado "dual deficit model" no que se refere à etiologia da psicopatia. Entretanto, o controle da variável ansiedade realizado a partir de uma análise complementar sugere que, em estudos mais amplos, pode ser adequado considerar uma menor manifestação de sintomas ansiosos em quadros de psicopatia propriamente ditos, considerando, para tanto, outras comorbidades. Faz-se necessário, nesse caso, mensurar manifestações de ansiedade diretamente relacionadas a traços da personalidade, com base na utilização de instrumentos elaborados e validados para esse fim. O presente estudo mensurou ansiedade utilizando um instrumento capaz de identificar sintomas mais recorrentes, porém não necessariamente atrelados a tendências comportamentais específicas que perfazem a personalidade.

De outro modo, a respeito da correlação entre fator 2 e sintomas depressivos e ansiosos, encontrada nesse trabaIho, a pesquisa de Stinson et al..$^{26}$ obteve resultados bastante semelhantes. As autoras investigaram a correlação entre os transtornos e descobriram que indivíduos que apresentaram sintomas mais recorrentes vinculados a transtorno de humor pontuavam mais no fator 2 do que no fator 1 da Escala Hare. Uma das hipóteses que se apresenta com base nos resultados obtidos é a de que tal relação sintomática pode ser identificada em níveis mais moderados, ainda que socialmente problemáticos, no que se refere à manifestação de comportamentos antissociais medidos pela Escala Hare. Dito de outro modo, é importante ressaltar que a amostra para o presente estudo foi composta por um número significativamente maior de indivíduos que não obtiveram pontuação suficiente para o diagnóstico de psicopatia. Esse fato demanda maior relativização no que se refere à possibilidade de comparação com trabalhos anteriores. Ao mesmo tempo, tais achados são, por si só, sugestivos quanto à necessidade de trabalhos mais amplos no Brasil, valendo-se de outros instrumentos ou mesmo de uma análise de classes latentes no que se refere à psicopatia, permitindo uma melhor investigação sobre a relação desta com diferentes transtornos de humor. Apesar dos resultados encontrados, Sareen et al. ${ }^{34}$ destacam que os profissionais que atuam na elaboração de políticas em saúde precisam considerar a coocorrência de transtornos de ansiedade e comportamentos antissociais. Essa relação não está circunscrita à manifestação da psicopatia, mas a uma série de tendências antissociais no sentido mais amplo da expressão.

\section{CONCLUSÕES}

Os achados deste trabalho permitiram observar que, embora alguns dados tenham sido concordantes com a literatura, por exemplo, a correlação entre sintomas depressivos e ansiosos, e a associação entre sintomas ansiosos e sintomas depressivos com o fator 2 da Escala Hare, os dados referentes a sintomas ansiosos e transtorno psicopático vão de encontro ao que a maior parte da literatura descreve. Além disso, ainda são poucos os estudos que encontram correlação entre transtorno psicopático e transtornos depressivos, não encontrando associação com sintomas ansiosos, como foi o caso desta pesquisa.

É necessário, ainda, destacar as limitações do estudo; uma dessas limitações diz respeito ao tamanho da amostra. Acredita-se que um número maior de participantes poderia resultar em dados mais consistentes. Outra limitação encontrada foi em relação aos instrumentos utilizados. Quanto às limitações das Escalas Beck (BDI e BAl), infere-se que, por não serem instrumentos de diagnóstico, somente foi possível oferecer especulações acerca dos sintomas afetivos em psicopatas, salientando-se, então, a importância da utilização de instrumentos que averiguassem profundamente a presença desses sintomas na população estudada. Além disso, utilizou-se o modelo de dois fatores do PCL-R, considerando que ele é condizente com as adaptações do instrumento feitas para a realidade brasileira, porém não mais com os novos estudos fatoriais com essa escala22.

Como principais contribuições, acredita-se que a presente pesquisa demonstra a importância de serem realizados mais estudos na área, a fim de possibilitar mais discussões sobre uma temática ainda pouco discutida no Brasil. Considera-se, ainda, que os resultados podem fomentar a realização de pesquisas com outros instrumentos e outras populações, oferecendo, assim, uma maior diversidade metodológica. 


\section{CONTRIBUIÇÕES INDIVIDUAIS}

Fernanda de Vargas - Mestranda responsável pela pesquisa que originou o presente artigo. Elaborou o projeto submetido à Instituição e ao Comitê de Ética. Realizou a coleta de dados, a análise e a interpretação dos resultados e escreveu o artigo, bem como aprovou sua versão final a ser publicada.

Fernanda Xavier Hoffmeister - Participou da coleta de dados, da análise e interpretação dos resultados, bem como colaborou com a elaboração do presente artigo e aprovou sua versão final a ser publicada.

Priscila Flores Prates - Participou da coleta de dados, da análise e interpretação dos resultados, bem como colaborou com a elaboração do presente artigo e aprovou sua versão final a ser publicada.

Silvio José Lemos Vasconcellos - Professor que orientou o projeto, a pesquisa, a análise e a interpretação dos dados. Revisou o conteúdo intelectual do artigo e aprovou sua versão final a ser publicada.

\section{CONFLITOS DE INTERESSE}

Fernanda de Vargas, Fernanda Xavier Hoffmeister, Priscila Flores Prates e Silvio José Lemos Vasconcellos não possuem conflitos de interesse a serem declarados.

\section{REFERÊNCIAS}

1. Sadock BJ, Sadock VA. Compêndio de psiquiatria: ciência do comportamento e psiquiatria clínica. 9a ed. Porto Alegre: Artmed; 2007.

2. American Psychiatric Association. Manual Diagnóstico e Estatístico de Transtornos Mentais: DSM-IV-TR. 4 a ed. Porto Alegre: Artmed; 2002.

3. Last CG, Perrin S, Hersen M, Kazdin AE. A prospective study of childhood anxiety disorders. J Am Acad Child Adolesc Psychiatry. 1996;35(11):1502-10.

4. Organização Mundial da Saúde. Salude mental: nuevos conecimientos, nuevas esperanzas. Informe sobre a saúde do mundo. Genebra: OMS; 2001.

5. Lima MS. Epidemiologia e impacto social. Rev Bras Psiquiatr. 1999;21(1):1-5.

6. Rodrigues MJSF. 0 diagnóstico de depressão. Psicologia USP. 2000;11(1):155-87.

7. American Psychiatric Association. Manual Diagnóstico e Estatístico de Transtornos Mentais: DSM -V. $5^{\text {a }}$ ed. Porto Alegre: Artmed; 2014.

8. Araújo FAFM, Nakano TC, Gouveia MLA. Prevalência de depressão e ansiedade em detentos. Aval Psicol. 2009;8(3):381-90.

9. Verona E, Hicks BM, Patrick CJ. Psychopathy and suicidality in female offenders: mediating influences of personality and abuse. J Consult Clin Psychol. 2005;73(6):1065-73.

10. Verona E, Patrick CJ, Joiner TE. Psychopathy, antisocial personality, and suicide risk. J Abnorm Psychol. 2001;110(3):462-70.
11. Lykken DT. A study of anxiety in the sociopathic personality. J Abnorm Psychol. 1957:55(1):6-10.

12. Patrick CJ, Bradley MM, Lang PJ. Emotion in the criminal psychopath: startle reflex modulation. J Abnorm Psychol. 1993;102(1):82-92.

13. Serafim AP, Barros DM, Valim A, Gorenstein C. Resposta cardíaca e nível de ansiedade em homicidas psicopatas. Rev Bras Psiquiatr. 2009;31(3):214-8.

14. Gray JA, Mcnaughton N. The neuropsychology of anxiety: an inquiry into the functions of the septo-hippocampal system. Oxford: Oxford University Press; 2000.

15. Creswell J. Projeto de pesquisa: métodos qualitativo, quantitativo e misto. $3^{\mathrm{a}}$ ed. Porto Alegre: Artmed; 2010.

16. Hulley SB, Cumming SR, Browner WS, Grady DG, Hearst NB, Newman TB. Delineando a pesquisa clínica: uma abordagem epidemiológica. 2ª ed. Porto Alegre: Artmed; 2003.

17. Amorim P. Mini International Neuropsychiatric Interview (MINI): validação de entrevista breve para diagnóstico de transtornos mentais. Rev Bras Psiquiatr. 2000;22(3):106-15.

18. Hare RD. The Hare Psychopathy Checklist Revised. 2a ed. Toronto, Canadá: Multi Health Systems; 2003

19. Morana HCP. Escala Hare PCL-R: critérios para pontuação de psicopatia revisados. Versão Brasileira. São Paulo: Casa do Psicólogo; 2004

20. Cunha JA. Manual da versão em português das Escalas Beck. São Paulo: Casa do Psicólogo; 2001.

21. Brasil. Ministério da Saúde. Resolução 466/12 do Conselho Nacional de Saúde/MS sobre Diretrizes e Normas Regulamentadoras de Pesquisa Envolvendo Seres Humanos. Diário Oficial da União; 2012

22. Hare RD, Harpur TJ, Hakstian AR, Forth AE, Hart SD. The revised Psychopathy Checklist: reliability and factor structure. Psychological Assessment. J Consult Clin Psychol. 1990;2(3):338-41.

23. Patrick CJ. Transtorno de personalidade antissocial e psicopatia. In: O'Donohue W, Fowler KA, Lilienfeld SO, editors. Transtornos de personalidade: em direção ao DSM-V. São Paulo: Roca; 2010.

24. Tavares GP, Scheffer M, Almeida RMM. Drogas, violência e aspectos emocionais em apenados. Psicol Reflex Crit. 2012;25(1):89-95.

25. Perez OM, Duque DVC, López SCA. Riesgo suicida y depresión en un grupo de internos de una cárcel del Quindío (Colombia). Investig Andina. 2011;13(23):268-80.

26. Stinson JD, Becker JV, Tromp S. A preliminary study on findings of psychopathy and affective disorders in adult sex offenders. Int J Law Psychiatry. 2005;28(6):637-49.

27. Gardner J, Bell A. Superando a ansiedade, o pânico e a depressão: novas maneiras de recuperar a autoconfiança. São Paulo: Madras; 2001.

28. Beck TA, Rush AJ, Shaw BF, Emery G. Terapia cognitiva da depressão. Rio de Janeiro: Zahar; 1982.

29. Fonseca KP. (Re)Pensando o crime como uma relação de antagonismo entre seus autores e a sociedade. Psicol Cienc Prof. 2006;26(4):532-47.

30. Baptista MN, Carneiro AM. Validade da escala de depressão: relação com ansiedade e stress laboral. Estud Psicol. 2011;28(3):345-52.

31. Barbosa GA, Gaião AA. Apontamentos em psicopatologia infantil. João Pessoa: Idéia; 2001.

32. Rodriquez-Sacristán J. Psicopatología del niño y de la adolescencia. Espanha: Universidad de Sevilla; 1995.

33. Torkelsen CN, Myklebust JJH. Psykopati og negativ affektivitet: Klarar CAPP å fange opp angst og depresjon i målinga av psykopati? [tese não publicada]. Noruega: Universitas Bergensis; 2013.

34. Sareen J, Stein MB, Cox BJ, Hassard ST. Understanding comorbidity of anxiety disorders with antisocial behavior: findings from two large community surveys. J Nerv Ment Dis. 2004;192(3):178-86. 